Der Hanns-Lilje-Preis 2008 wurde Herrn Andres Straßberger, Grossbothen, für seine Arbeit "Johann Christoph Gottsched und die ,Philosophische' Predigt. Studien zur aufklärerischen Transformation der protestantischen Homiletik im Spannungsfeld von Theologie, Philosophie, Rhetorik und Politik" verliehen.

\title{
Johann Christoph Gottsched (1700-1766) und die „philosophische“ Predigt: Studien zur aufklärerischen Transformation der protestantischen Homiletik im Spannungsfeld von Theologie, Philosophie, Rhetorik und Politik
}

\section{Andres Strassberger}

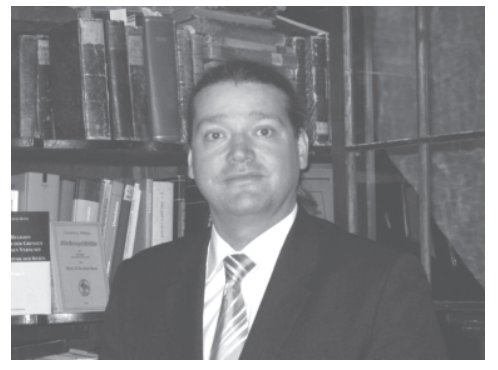

Andres Straßberger, Geschäftsführer des Landeskirchlichen Prüfungsamtes der Evangelisch-Lutherischen Landeskirche Sachsens in Leipzig, Träger des Hanns-Lilje-Preises 2008
Die forschungsstrategische Relevanz einer kirchenhistorischen Arbeit wie der meinigen lässt sich vor einem gemischten Publikum wie dem gegenwärtigen auf verschiedene Weise erläutern. Das Stichwort der ,aufklärerischen Transformation", wie es in der Titelformulierung meiner Dissertation begegnet, verweist auf einen retrospektiv gesehen - epochalen geschichtlichen Wandlungsprozess, der das Erscheinungsbild des Christentums im allgemeinen und des Protestantismus im besonderen so nachhaltig verändert hat, dass seine vor- und seine nachaufklärerische Gestalt sich nur noch bedingt miteinander gleichsetzen lassen können. Doch ist das Erbe der Aufklärung nicht nur in der Gegenwart andauernd präsent, sondern es ist zugleich innertheologisch umstritten wie kaum ein anderes Resultat der Kirchengeschichte. Ein konkretes Beispiel aus der jüngsten Zeit möge das illustrieren.

Vor wenigen Wochen fand in Chemnitz mit „Pro Christ“ eine mehrtägige Missionsveranstaltung nach amerikanischem Vorbild statt. Das medial und innerkirchlich mit viel Begleitmusik inszenierte Großereignis fand nach seinem Abschluss in der sächsischen Kirchenzeitung einen der bei solchen Gelegenheiten regelmäßig auftretenden Kritiker. Dieser, selbst ein 
Chemnitzer, warf den Veranstaltern und Unterstützern des Events in einem Leserbrief vor, die Kirche als „marktschreierische und zugleich hinterwäldlerische Evangelikalenbewegung" (Der Sonntag: Wochenzeitung für die Evangelisch-Lutherische Landeskirche Sachsens, Nr. 16, 19.4. 2009, S. 9) präsentiert zu haben. Dem Hauptprediger der Veranstaltung warf er vor, ein „Selbstdarsteller" und „evangelikaler Tendenzprediger mit fundamentalistischen Ansichten" (beide Zitate ebd.) zu sein. Sich selbst bezeichnete er dagegen als „liberalen Christen“, für den „Bibelkritik, die Segnung Homosexueller und die Evolutionslehre Darwins keine Fremdworte sind" (beide Zitate ebd.). Diese Wortmeldung rief eine Flut von Gegenbriefen hervor, die nicht nur den polemischen Tonfall monierten. Ein Dresdner Leserbriefschreiber hielt dem Verfasser des Leserbriefs entgegen (Der Sonntag, Nr. 18, 3.5.2009, S. 9): „Die liberale, bibelkritische Theologie ist der Tod der Kirche. [...] Die sogenannte moderne Theologie in Folge der Aufklärung ist der zweite Sündenfall der Menschheit. Ja schlimmer noch, es ist der Sündenfall der Christenheit [... “"

Die Aufklärung - der zweite Sündenfall der Christenheit: pointierter kann man die Abneigung, ja religiös motivierte Gegnerschaft zu einer ganz bestimmten Epoche der abendländischen Geistes-, Kultur- und damit auch Kirchengeschichte nicht auf den Punkt bringen. Dabei ist vorauszusetzen, dass es sich im vorliegenden Fall keineswegs um eine besonders extreme Einzelmeinung handelt, sondern um die Sicht eines ganz bestimmten religiösen Milieus. Mein historiographisches Interesse an der theologischen Aufklärung ist auf diesem Hintergrund zu verstehen, und das Thema meiner Arbeit gewinnt bei allen rein historischen Fragestellungen in dieser Perspektive ihr letzthin gegenwartstheologisches Profil. Denn als ein dem theologischen Erbe der Aufklärung prinzipiell positiv gegenüberstehender Theologe und Historiker möchte ich über die - je nach theologischem Standpunkt - identifikatorisch beanspruchte oder verunglimpfte Epoche europäischer Kirchen- und Kulturgeschichte im Medium der Geschichtsschreibung zunächst erst einmal aufklären. Daher zielt meine Dissertation im Kern darauf, die gegenwärtige Umstrittenheit der Aufklärung historisch zu distanzieren, d.h. die Epoche vor jeder positiven oder negativen Inanspruchnahme zunächst erst einmal einer möglichst sachlichen, differenzierten historischen Beschreibung und Beurteilung zu unterziehen.

Dass ich dafür nicht „die Aufklärung“ per se und in toto zum Untersuchungsgegenstand machen konnte, lag und liegt auf der Hand. So gingen in der Konzeptionsphase meine Überlegungen dahin, den Versuch zu unternehmen, an einem konkreten Fallbeispiel nachzuzeichnen, wie sich der Prozess der Aufklärung in der Theologie vollzogen hat, und zwar nicht 
allein als rein ideengeschichtlicher, sondern auch als praktischer, von einer Vielzahl von konkreten Menschen getragener. Von entscheidender Bedeutung für den Erfolg dieses Unternehmens sollte sich die Fokussierung und Konkretisierung des Untersuchungsgegenstands erweisen.

Es war mein im Jahr 2001 leider viel zu früh verstorbener erster Doktorvater Kurt Nowak, der an diesem Punkt der Projektkonzeption meinen Blick auf den theologie- und kirchengeschichtlich bis dahin völlig ignorierten Leipziger Philosophieprofessor und Literaturreformer Johann Christoph Gottsched lenkte (vgl. Abbildung 1). Ich kann an dieser Stelle offen zugeben, dass er dies nicht gerade zu meiner ausgesprochenen Freude tat. Denn aufgrund einer bildungsbürgerlichen Prädisposition durch mein Elternhaus (meine Mutter pflegte als studierte Germanistin des öfteren mit Wohlgefallen das ebenso souveräne wie historisch ungerechte Verdikt Lessings über Gottsched im 17. Literaturbriefzu zitieren) bzw. durch eine Lektüre von Goethes „Dichtung und Wahrheit“, worin der Leipziger Literaturpapst als „präzeptorialer Perückenständer“ (R. Wittmann) karikiert und damit meinem schallenden Gelächter preisgegeben wurde, vermochte ich dem in wolffisch-philosophischem Vernunftrationalismus durchaus verknöcherten Aufklärer nicht jene Begeisterung abzugewinnen, die ich mir

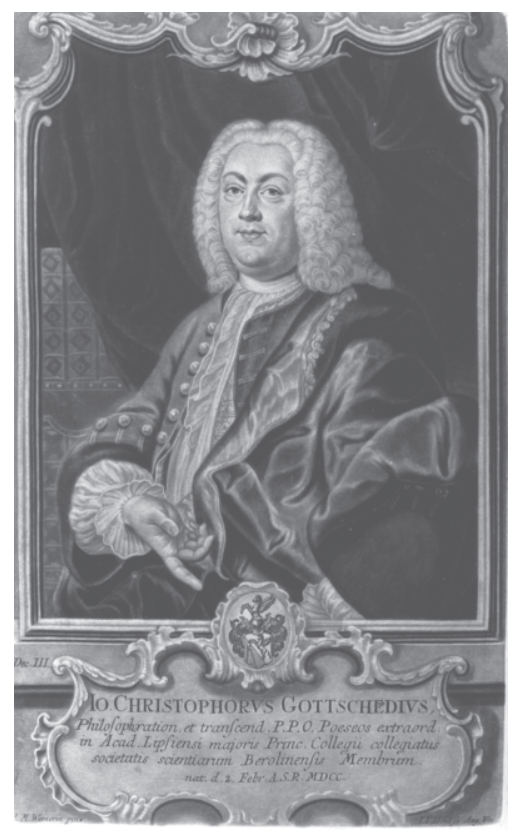

Abbildung 1: Porträt J. C. Gottscheds 
vom Helden meiner Dissertation eigentlich gewünscht hatte. Bei aller inneren Distanz zu Gottsched, die auch bis zuletzt geblieben ist, hatte ich nach einer kurzen Zeit der Einarbeitung jedoch nicht mehr den geringsten Zweifel daran, dass dieser eine für den Aufklärungsprozess ungeheuer dynamisierende Rolle gespielt hat, die mehr als nur eine kirchenhistorische Arbeit rechtfertigte.

Da mir in dieser Phase des Projekts nicht nur ein von Gottsched anonym publiziertes Predigtlehrbuch in die Hände fiel, sondern auch weiteres, die aufklärerische Predigtreform betreffendes Material seines Briefwechsels und anderer Quellen, z.B. kirchliche Verhörakten im Zusammenhang mit seiner reformhomiletischen Publikationstätigkeit, überlegte ich, ob es nicht möglich sein könnte, die übergeordnete Frage nach dem Prozess der theologischen Aufklärung anhand von Gottscheds Beteiligung an der aufklärerischen Homiletikreform zu exemplifizieren. Dass der Fokus neben dem Leipziger Aufklärer dabei in für einen protestantischen Kirchenhistoriker willkommener Weise auf die Geschichte der Predigt bzw. ihrer Theorie fallen sollte, bedarf zumindest für denjenigen keiner weiteren Begründung, der eine auch nur vage Vorstellung von der theologischen und frömmigkeitsgeschichtlichen Stellung der Predigt in den Kirchen der Reformation hat.

Nach einer nun folgenden extensiven Recherche, in der versucht wurde, den gedruckten Quellenbestand zur Predigtreform im zweiten Viertel des 18. Jahrhunderts sowie ausgewähltes Material der archivalischen Überlieferung so vollständig wie nur möglich zu erfassen und auszuwerten, blieb vor allem zu klären, wie sich die dabei bestätigte aufklärungspropagandistische Rolle Gottscheds im predigttheoretischen und -praktischen Kontext angemessen zur Darstellung bringen lassen könnte. Angesichts der Mehrdimensionalität des Quellenmaterials und der Interdisziplinarität der Fragestellung entschied ich mich für ein multiperspektvisches Vorgehen. Unter wechselnden methodischen Gesichtspunkten und Fragestellungen hoffte ich, mittels einer Reihe von aufeinander bezogenen und miteinander in Zusammenhang stehenden Teilstudien Antworten auf meine übergeordnete Leitfrage - Was war homiletische Aufklärung bzw. wie vollzog sie sich? - zu erhalten.

In einem ersten Schritt ging es mir darum, die biographischen und die geistigen Gelenkstellen für Gottscheds homiletisches Reformstreben ausfindig zu machen. Denn sein Weg führte ihn von einem Königsberger Theologiestudium in eine Leipziger Poetik- und Philosophieprofessur, in welcher letzteren Funktion er dann auch zum anonymen Lehrer der Homiletik wurde. Anders gesagt, es ging mir im ersten Kapitel darum, Gottscheds biographische Synthese von Theologie, Philosophie und Literatur als her- 
meneutischen Hintergrund seines reformhomiletischen Handelns transparent zu machen. Dabei rechtfertigte es das Exemplarische seiner Biographie, das den ostpreußischen Pfarrerssohn als paradigmatischen Vertreter derjenigen Generation von Studenten und Gelehrten erscheinen lässt, die Christian Wolffs Philosophie zum Leitstern ihrer wissenschaftlichen Aktivitäten machten, den angedeuteten Fragen ein historiographisches Eigenrecht zuzubilligen. Dabei wurde u.a. deutlich, dass sein Bruch mit der Theologie und seine Hinwendung zur Philosophie kein Bruch mit dem Christentum an und für sich war, sondern lediglich eine Abkehr von deren konservativkirchlicher Entwicklungsgestalt. In frömmigkeitsgeschichtlicher Perspektive begegnet uns mit Gottsched daher ein Vertreter des aufkommenden Aufklärungschristentums, als der er auch in den homiletischen Diskurs seiner Zeit eingriff.

Das zweite Kapitel widmete sich dann detailliert Gottscheds Theorie einer vernunftaufklärerisch reformierten Predigt. Nach ersten Überlegungen der 1720er Jahre kulmilierte seine diesbezügliche Programmatik 1736 in einem die Predigt betreffenden Kapitel der „Ausführlichen Redekunst“ sowie dem 1740 anonym publizierten „Grund=Riß einer Lehr=Arth ordentlich und erbaulich zu predigen" (2. Auflage 1743; Abbildung 2). In die-
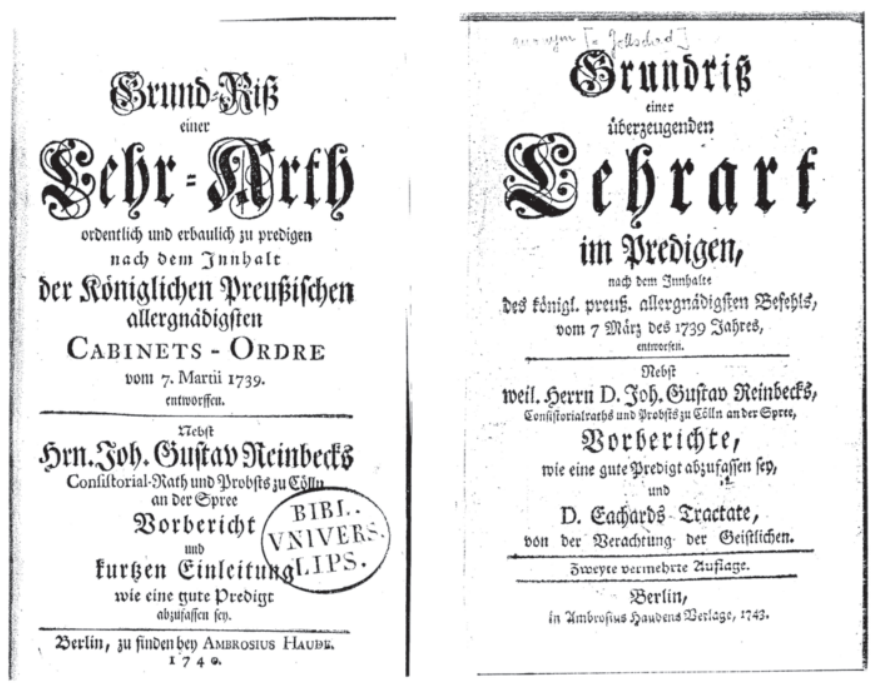

Abbildung 2: Titelblätter der ersten und der zweiten Auflage von Gottscheds Homiletik (Exemplare der Universitätsbibliothek Leipzig bzw. der Stiftsbibliothek Kremsmünster/ Österreich) 
ser Teilstudie, die in intensiver Weise das Gespräch mit der bisherigen literaturwissenschaftlichen, insbesondere rhetorikgeschichtlichen, sowie philosophiegeschichtlichen Gottsched- bzw. Wolffforschung suchte, konnte aufgezeigt werden, warum und wie sich für Gottsched aus einer Reform des Denkens (Philosophie) zunächst eine Reform der Sprache und Rede (Rhetorik) und, als Sonderfall darin eingelagert, eine Reform der Predigt (Homiletik) ergab. Diese Systematik, die die Homiletik in starke Abhängigkeit von verschiedenen Prämissen der Wolffschen Philosophie brachte, führte im Ergebnis zu einer homiletischen Konzeption, die bereits zeitgenössisch das Etikett einer „philosophischen Predigt“ erhielt, was ich in der Formulierung meines Dissertationsthemas aufgriff.

In der dritten Teilstudie wurde schließlich der Versuch unternommen, die Propagierung von Gottscheds Homiletikreform durch seine Anhänger an wichtigen Schaltstellen aufzuhellen. Die diesbezüglichen Fragestellungen wurden dabei in einer sozietätsgeschichtlichen Perspektive gebündelt. In den Blickpunkt kamen hier die von Gottsched 1727 reformierte und bis zu seinem Austritt 1738 als Senior geführte „Deutsche Gesellschaft" zu Leipzig, sodann zwei von ihm um 1730 gegründete Rednergesellschaften sowie die von Ernst Christoph Graf von Manteuffel 1736 ins Leben gerufene Alethophilengesellschaft, in welcher Gottscheds Predigtreformprogramm den Mittelpunkt der Sozietätsaktivitäten bildete. In diesem Zusammenhang wurden auch die politischen Implikationen von Gottscheds Predigtlehrbuch untersucht, wie sie in dessen titelgebendem Bezug auf eine preußische Kabinettsorder von 1739 gegeben war. Insgesamt wurde mit der in den genannten Sozietäten verankerten homiletischen Propaganda des Gottsched-Kreises ein zentrales positives Konstitutionsmerkmal des „Kommunikationsprozesses der homiletischen Aufklärung“ thematisiert, das in der vierten Teilstudie ihr negatives Komplement erhielt. Denn als zweites entscheidendes Moment analysierte ich als nächstes den öffentlichen, ganz überwiegend in deutscher Sprache ausgetragenen Streit um Gottscheds Predigtreformprogramm bzw. die „philosophische“ Predigt. Die hierfür im Anschluss an und in Analogie zu Martin Gierl vorausgesetzte These lautete, dass der Prozess der homiletischen Aufklärung sich wesentlich erst durch jenen Streit konstituiert habe, der um sie geführt wurde. Die Gegner des homiletischen Wolffianismus wurden in diesem Zusammenhang nach einer theologiegeschichtlich üblichen Differenzierung separat unter die Lupe genommen: die aristotelisch beeinflusste Spätorthodoxie, der von der "philosophia eclectica“ partiell beeinflusste Pietismus sowie die - ebenfalls „eklektisch“ eingestellte - sogenannte „Übergangstheologie“. In diesem Zusammenhang kamen in einem eigenen Teilkapitel auch 
einige Gottsched-Kritiker aus den Reihen der 1737 gegründeten Universität Göttingen in den Blick, als deren profiliertester der heute in der Theologieund der Kirchengeschichtsschreibung weithin vergessene Joachim Oporin angesehen werden kann. Die Analyse des ganz überwiegend mit Mitteln der Publizistik geführten Streits zeigte, dass sich entlang der Kampflinie verschiedene Positionen im innertheologischen Umgang mit dem Vernunftanspruch der Aufklärung organisierten, die in ihrer jeweiligen Argumentationslogik bis heute gängige Reaktionsmuster präfigurierten.

Im fünften und letzten Kapitel kam schließlich die Krise der „philosophischen" Predigt um 1750 in den Blick, die in einen gesamtkulturellen Bedeutungsverlust der Wolffschen Philosophie eingebettet war. Dazu wandte ich mich zunächst der von Georg Friedrich Meier, dem Halleschen Philosophieprofessor, vorgeschlagenen Alternative zu, „ästhetisch“ zu predigen. Seine im Gefolge von Weiterentwicklungen der Erkenntnistheorie Wolffs stehende homiletische Konzeption war aber nur ein Ansatz der von mehreren Seiten gleichzeitig in Angriff genommenen Reformbemühungen, die allenthalben darauf zielten, rationalistische Engführungen der „philosophischen " Predigt aufzubrechen. Eine stärker theologisch motivierte Alternative, die Konzeption der „moralischen“ Predigt, wie sie von Theologen wie August Friedrich Wilhelm Sack und Johann Joachim Spalding vertreten wurde, wies deshalb - trotz eigener ideengeschichtlicher Anschlussstellen Gemeinsamkeiten mit Meiers Ansatz auf. Der bis dahin erreichte Grundkonsens der „philosophischen“ Predigt, nämlich die an Vernunftkriterien orientierte Verstandesaufklärung zur Voraussetzung eines primär intendierten Glaubensaffektes in der Predigt zu machen, wurde dabei jedoch zu keinem Zeitpunkt aufgekündigt. Dies sollte erst zu Beginn des 19. Jahrhunderts mit Schleiermacher erfolgen. Rückblickend geurteilt, hat allerdings Gottscheds Auffassung, dass eine Predigt als Rede den „vernünftigen" Regeln der antiken Rhetorik zu folgen habe, - eine Ansicht, die bei ihm gegen barockrhetorisches Geschmacksempfinden gerichtet war - bis heute nichts an grundsätzlicher Plausibilität eingebüßt. So gesehen, beginnt in homiletikgeschichtlicher Perspektive mit Gottsched die Geschichte der modernen Predigt, für die er das erste Lehrbuch geschrieben hat. 